\title{
Morfología Ósea Facial en Cirugía Ortognática. ¿Existe Tendencia Hacia el Avance Facial?
}

\author{
Facial Morphology in Orthognathic Surgery. Is There a Facial Advance Trend?
}

\author{
Víctor Ravelo ${ }^{1}$; Gabriela Olate ${ }^{1}$; Claudio Huentequeo-Molina²; Ziyad S. Haidar ${ }^{3,4}$; \\ Marcelo Parra ${ }^{1,5}$; Bélgica Vásquez ${ }^{6} \&$ Sergio Olate $^{1,2,5}$
}

RAVELO, V.; OLATE, G.; HUENTEQUEO-MOLINA, C.; HAIDAR, Z. S.; PARRA, M.; VÁSQUEZ, B. \& OLATE, S. Morfología ósea facial en cirugía ortognática. ¿Existe tendencia hacia el avance facial?. Int. J. Morphol., 39(4):1116-1122, 2021.

RESUMEN: El objetivo de esta investigación fue determinar los movimientos preferidos en maxila y mandíbula para obtener normalidad en morfología facial utilizando técnicas de superimposición en análisis 3D. Se realizó un estudio descriptivo para evaluar el desplazamiento óseo bimaxilar y del hueso hioides en sujetos clase facial tipo II y clase facial tipo III sometidos a cirugía ortognática. Para la superimposición se utilizó como puntos fijos Nasion - Silla - Porion y la sutura cigomática-maxilar. Estos puntos se superpusieron en CBCT pre quirúrgico y postquirúrgico y se evaluó el desplazamiento de la espina nasal anterior, Punto A, Punto B, mentón y del hueso hioides. Para la evaluación y comparación de las variables continuas antes y después de la cirugía ortognática se utilizó la prueba $\mathrm{T}$ de Student. Para la correlación entre las variables, se utilizó el Test de Spearman considerando un valor p $<0,05$ como diferencia significativa. 44 sujetos de entre 18 y 40 años de ambos sexos, fueron incluidos en esta investigación. En el $90 \%$ de los sujetos se realizó un movimiento sagital de avance de la maxila. El movimiento sagital de avance mandibular se realizó en el $100 \%$ de los sujetos con clase facial tipo II, mientras que el $100 \%$ de los sujetos con clase facial tipo III se realizó se le retroceso mandibular. El hueso hioides presentó un avance en 26 de los 27 sujetos con clase facial tipo III. Es posible concluir que existe una tendencia al avance maxilar independiente de la deformidad facial.

KEY WORDS: Cirugía ortognática; Morfología facial; Cefalometría; Movimiento óseo.

\section{INTRODUCCIÓN}

Las deformidades faciales son un complejo grupo de alteraciones faciales asociadas a defectos del desarrollo del esqueleto facial. En particular, la maxila y la mandíbula son afectados por un crecimiento que puede ser deficiente por condiciones hereditarias y por condiciones ambientales (Goulart et al., 2018).

La maxila es el hueso que forma el tercio medio facial. Es un hueso que sustenta el proceso alveolar que soporta los dientes, forma la pirámide nasal, el piso y contorno orbitario y se relaciona con el hueso cigomático para entregar la forma lateral del rostro (Villa et al., 2020; Ravelo et al., 2021a). La mandíbula, por otra parte, desarrolla todo el tercio inferior del rostro, de forma que la posición de la lengua, labios, área submandibular, vía aérea y contorno facial inferior son relacionados con la posición de la mandíbula (Ravelo et al., 2020a; Ravelo et al., 2021b).

La posición de la maxila y mandíbula son fundamentales también para el soporte de los músculos faciales y determinar la posición que ellos tienen a lo largo de la vida (Shaw et al., 2012); de la misma forma, los paquetes de grasa facial y el soporte de ellos sobre la estructura ósea y muscular son vinculados a la estructura ósea maxilar y mandibular (Sykes et al., 2020).

Recientes estudios, además, han indicado que el envejecimiento facial es vinculado a remodelaciones y cam-

\footnotetext{
${ }^{1}$ Centro de Excelencia en Estudios Morfológicos y Quirúrgicos, Universidad de La Frontera, Temuco, Chile.

${ }^{2}$ Departamento de Cirugía Oral y Maxilofacial, Hospital AGP, Lautaro, Chile.

${ }^{3}$ BioMAT'X, Facultad de Odontología, Universidad de los Andes, Santiago, Chile.

${ }^{4}$ Centro de Investigación e Innovación Biomédica (CIIB), Facultad de Medicina, Universidad de los Andes, Santiago, Chile.

${ }^{5}$ División de Cirugía Oral, Facial y Maxilofacial, Universidad de La Frontera, Temuco, Chile.

${ }^{6}$ Facultad de Ciencias de la Salud, Universidad de Tarapacá, Iquique, Chile.
} 
bios en la posición de la maxila y mandíbula (Medelson \& Wong, 2012), de forma que mantener una condición alterada de estos huesos implica cambios en el modelo de envejecimiento facial acentuando algunos efectos de este proceso.

Las técnicas quirúrgicas en cirugía ortognática se han desarrollado eficientemente en los últimos 60 años (Obwegeser, 2007). Técnicas contemporáneas de cirugía ortognática con diferentes aproximaciones, accesos, técnicas de osteotomía y fijación han sido desarrolladas con bajas complicaciones (Olate et al., 2018).

El objetivo de esta investigación fue determinar los movimientos preferidos en maxila y mandíbula para obtener normalidad en morfología facial utilizando técnicas de superimposición en análisis 3D.

\section{MATERIAL Y MÉTODO}

Se realizó un estudio descriptivo para evaluar el desplazamiento óseo bimaxilar y del hueso hioides en sujetos clase facial tipo II y clase facial tipo III sometidos a cirugía ortognática. Fueron incluidos 44 sujetos entre 18 y 40 años de edad, de ambos sexos con algún tipo de deformidad facial; se realizó una evaluación sagital utilizando el análisis de Steiner para determinar la clase esqueletal, donde los sujetos que presentaron un ángulo $>4^{\circ}$ fueron considerados clase II esqueletal y los sujetos que presentaron un ángulo $<0^{\circ}$ fueron considerados clase III esqueletal. Fueron excluidos sujetos con cirugía ortognática previa, antecedentes de trauma facial, presencia de síndromes y sujetos con asimetría facial con una desviación del mentón mayor a $5 \mathrm{~mm}$ desde la línea media facial. Los sujetos que participaron en este estudio firmaron un documento de consentimiento informado, y la investigación se realizó resguardando la integridad de los participantes y respetando la Declaración de Helsinki.

Cirugía. El plan quirúrgico de cada sujeto fue realizado mediante estudio y análisis tridimensional. El plan quirúrgico se basó en un análisis clínico facial de proporciones y posiciones de tejidos blandos faciales, un análisis de imagen 2D y 3D que determina la posición de los huesos maxilar y mandíbula según parámetros de estudios cefalométricos útiles para cada caso (REF) y, un estudio de factibilidad de movimientos en base a la oclusión dental de cada paciente. Todos los sujetos utilizaron ortodoncia previa a la cirugía ortognática. Luego que el plan quirúrgico fue aprobado, la impresión 3D fue utilizada para imprimir guías de corte y posicionamiento maxilar según cada caso.
La cirugía fue realizada utilizando anestesia general. Todas las osteotomías fueron realizadas con sistemas piezoeléctrico (Satelec, Action, Francia). En la maxila se utilizó la osteotomía de Le Fort I (LFI) mediante acceso intraoral según la técnica previamente publicada por Olate et al. (2014); todos los casos fueron fijados con 4 placas de osteosíntesis del sistema 2.0

En la mandíbula fue utilizada la técnica de osteotomía sagital de rama mandibular (OSRM) mediante acceso intraoral según la técnica previamente publicada. En todos los casos la osteotomía fue estabilizada con placas de osteosíntesis del sistema 2.0 con tornillos monocorticales.

Para cada caso, fueron analizados movimientos de maxila y mandíbula considerando los movimientos tridimensionales de tipo sagital, vertical y transversal incluyendo movimientos monomaxilares y bimaxilares.

Análisis de Morfología por Superimposición. Todos los sujetos fueron estudiados en la etapa preoperatoria con una tomografía computadorizada de haz cónico (CBCT) para realizar, el análisis y planificación virtual. El estudio se desarrollo en el equipo 3D NewTom, Modelo VGi EVO (Verona Italia), campo de visualización de 24 X $19 \mathrm{~cm}$ y parámetros de exposición 110 $\mathrm{kV}, 8 \mathrm{~mA}, 15 \mathrm{seg}$. El mismo estudio fue realizado una semana previo a la intervención y 30 días después de la cirugía.

Para el proceso de superimposición se utilizó como puntos fijos Nasion (N) - Silla (S) - Porion (Po) y la sutura cigomática-maxilar (Z). Estos puntos se superpusieron en CBCT pre quirúrgico y postquirúrgico y se evaluó el desplazamiento de la espina nasal anterior (ENA), Punto A, Punto B, Mentón (Me) y del hueso hioides (Figs. 1 y Fig. 2).

Análisis estadístico. Las mediciones fueron realizadas por el mismo observador en distintos tiempos, con diferencia de dos semanas entre los 44 estudios. Para las variables continuas se obtuvo un índice intraclase de 0,84 . El análisis de los datos se realizó con el software Graph Prism versión 9.1.0. Los parámetros clínicos se presentaron como media y desviación estándar. Para el análisis de distribución normal se utilizo la prueba de Shapiro-Wilk. Para la evaluación y comparación de las variables continuas antes y después de la cirugía ortognática se utilizó la prueba T de Student. Para la correlación entre las variables, se utilizó el Test de Spearman considerando un valor $\mathrm{p}<0,05$ como diferencia significativa. 


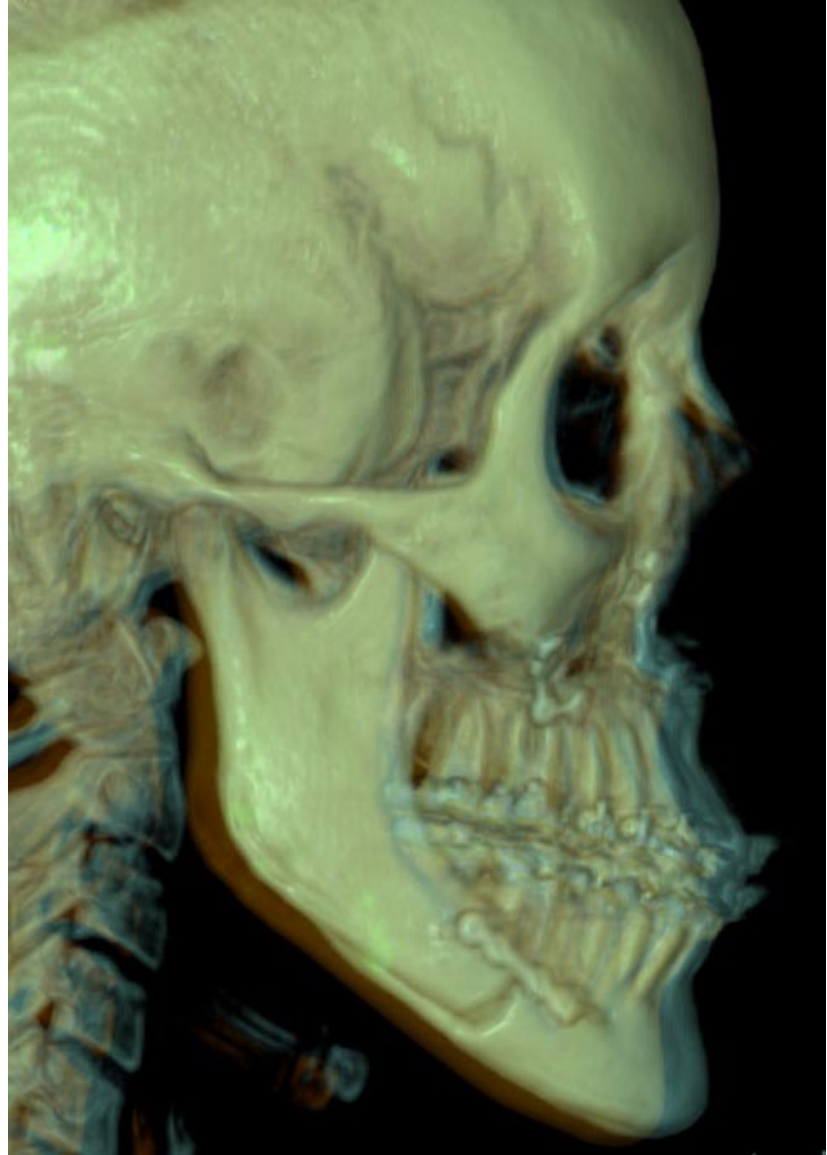

Fig. 1. Superimposición en sujeto de clase II facial.

\section{RESULTADOS}

Del total de sujetos evaluados (44), 27 (61,4\%) fueron de clase II esqueletal y $17(38,6 \%)$ fueron de clase III. La cirugía bimaxilar se realizó en 40 (90,1\%) de los 44 sujetos intervenidos.

Los movimientos quirúrgicos más usuales fueron de avance mandibular en el 14,8\% de los sujetos clase II y avance bimaxilar en el 85,2\% de los sujetos clase II; para los sujetos clase III se produjo un movimiento de avance maxilar y retroceso mandibular.

Respecto del análisis de movimiento del tercio medio facial, en el $90 \%$ de los sujetos se realizó un movimiento sagital de avance de la maxila; el movimiento vertical fue de ascenso con 29 sujetos $(65,1 \%)$ y de descenso en $11(25 \%)$ de los sujetos, sin diferencias significativas entre ellos $(\mathrm{p}=0,71)$.

En el análisis de movimiento del tercio inferior facial, el movimiento sagital de avance mandibular se rea-

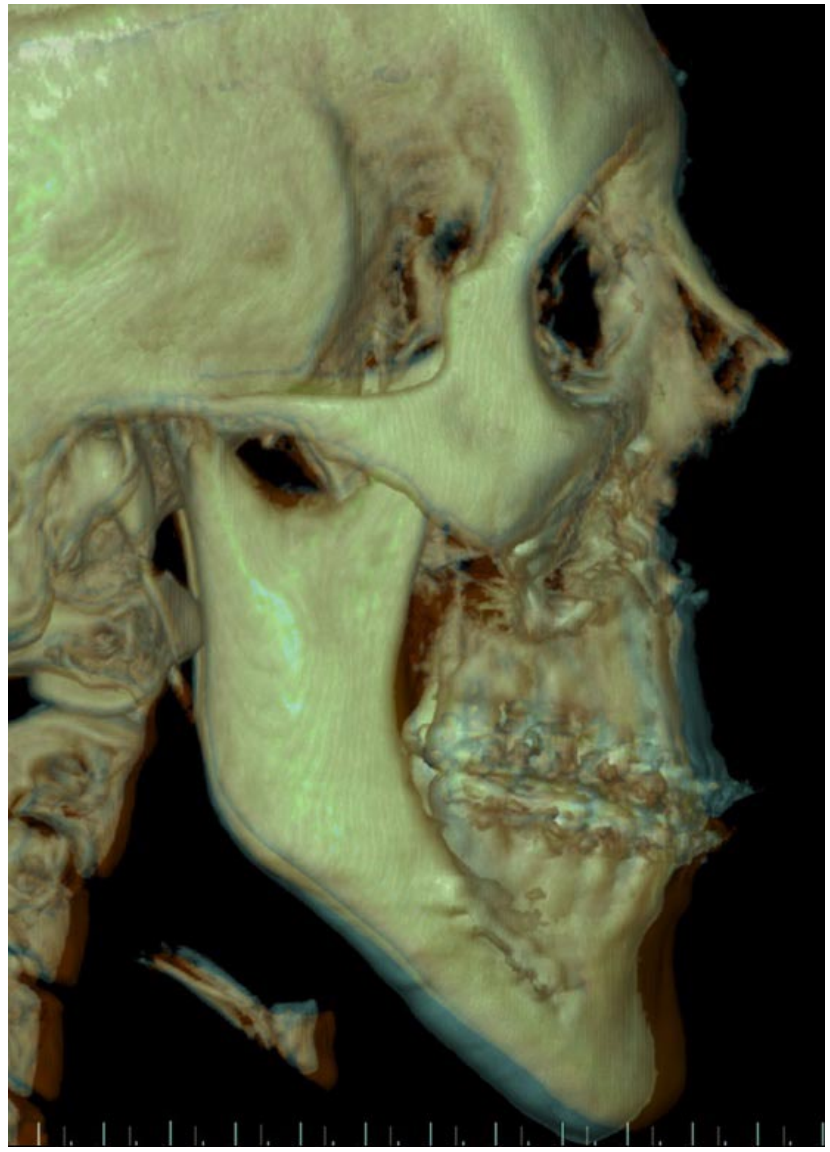

Fig. 2. Superimposición en sujeto de clase III facial.

lizó en el $100 \%$ de los sujetos con diagnóstico de clase facial tipo II, mientras que fue de retroceso en el $100 \%$ de los sujetos con diagnóstico de clase facial tipo III. El movimiento vertical fue de reposición superior en $26 \mathrm{su}-$ jetos $(59,1 \%)$ y de reposición inferior en 18 (40,9\%), sujetos con diferencias significativas entre ellos $(\mathrm{p}=0,025)$ (Tabla I).

El hueso hioides presentó movimiento de avance en 26 de los 27 sujetos con clase II esqueletal y cirugía de avance mono o bimaxilar; en el caso de los sujetos clase III esqueletal, quienes fueron tratados frecuentemente con retroceso mandibular, se presentó un retroceso del hueso hioides en 13 de los 17 pacientes operados. En el análisis general, la tendencia fue significativamente superior $(\mathrm{p}=0,0001)$ para el avance del hueso hioides en el 68,2\% como resultado del movimiento. En el patrón vertical, el hueso hioides presentó un ascenso en 31 (70,5\%) sujetos, siendo significativamente mayor que las situaciones de descenso del hueso hioides (Tabla II). 
En términos de magnitud del avance, el movimiento sagital del punto A fue de $2,07 \mathrm{~mm}( \pm 1,24)$ en los sujetos clase II y de $3,59 \mathrm{~mm}( \pm 1,31)$ en los sujetos clase III. El punto B presentó un movimiento sagital de avance de 4,37 $\mathrm{mm}( \pm 1,25)$ para los sujetos clase II y $3,68 \mathrm{~mm}( \pm 1,24)$ de retroceso en los sujetos clase III. Todos los movimientos sagitales presentaron diferencias significativas al comparar los sujetos clase II y clase III esqueletal (Tabla III).

El punto ENA presentó un promedio de ascenso de casi $1 \mathrm{~mm}$ para ambos grupos, sin diferencias; sin embargo, el punto Me presentó ascenso de $1,18 \mathrm{~mm}( \pm 2,69)$ para los sujetos clase II esqueletal y de $0,81 \mathrm{~mm}( \pm 3,04)$ de descenso en los sujetos clase III esqueletal.

Tabla I. Distribución de sujetos según la clase esqueletal y la prevalencia del movimiento maxilomandibular post cirugía ortognática.

\begin{tabular}{|c|c|c|c|c|c|}
\hline & \multicolumn{2}{|c|}{ Clase esqueletal tipo II } & \multicolumn{2}{|c|}{ Clase esqueletal tipo III } & \multirow[b]{2}{*}{$\mathbf{P}$} \\
\hline & $\mathbf{N}$ & $\%$ & $\mathbf{N}$ & $\%$ & \\
\hline \multicolumn{6}{|l|}{ Sagital } \\
\hline Avance Mx & 23 & 52,27 & 17 & 38,63 & $0,0005 *$ \\
\hline Ausencia de movimiento $\mathrm{Mx}$ & 4 & 9,09 & 0 & 0 & \\
\hline Retroceso Mx & 0 & 0 & 0 & 0 & \\
\hline \multicolumn{6}{|l|}{ Vertical } \\
\hline Ascenso Mx & 17 & 38,63 & 12 & 27,27 & 0,71 \\
\hline Descenso Mx & 6 & 13,63 & 5 & 11,36 & \\
\hline Ausencia de movimiento $\mathrm{Mx}$ & 4 & 9,09 & 0 & 0 & \\
\hline \multicolumn{6}{|l|}{ Sagital } \\
\hline Avance Mn & 27 & 61,13 & 0 & 0 & $0,0001 *$ \\
\hline Retroceso Mn & 0 & 0 & 17 & 38,63 & \\
\hline \multicolumn{6}{|l|}{ Vertical } \\
\hline Ascenso Mn & 19 & 43,18 & 7 & 15,90 & $0,025^{*}$ \\
\hline Descenso Mn & 8 & 18,18 & 10 & 22,72 & \\
\hline
\end{tabular}

Mx: maxilar; Mn: mandíbula; N: corresponde al número de individuos para cada característica y se encuentra expresado en porcentajes en la fila lateral (\%). (*) indica diferencia estadística significativa.

Tabla II. Distribución de sujetos según la clase esqueletal y la prevalencia del movimiento del hueso hioides post cirugía ortognática.

\begin{tabular}{lccccc}
\hline & Clase esqueletal tipo II & \multicolumn{3}{l}{ Clase esqueletal tipo III } & \\
\hline Sagital & $\mathbf{N}$ & $\%$ & $\mathbf{N}$ & $\%$ & $\mathbf{P}$ \\
Avance Hioides & 26 & 59,09 & 4 & 9.09 & $0,0001^{*}$ \\
Retroceso Hioides & 1 & 2,27 & 13 & 29,54 & \\
Vertical & & & & & \\
Ascenso Hioides & 25 & 56,81 & 6 & 13,63 & $0,002^{*}$ \\
Descenso Hioides & 2 & 4,54 & 11 & 25,00 & \\
\hline
\end{tabular}

$\mathrm{N}$ : corresponde al número de individuos para cada característica y se encuentra expresado en porcentajes en la fila lateral $(\%) .(*)$ indica diferencia estadística significativa. 
Tabla III. Distribución de sujetos según la clase esqueletal y la diferencia de movimiento transversal y vertical post cirugía ortognática.

\begin{tabular}{|c|c|c|c|c|c|}
\hline & \multicolumn{2}{|c|}{ Clase esqueletal tipo II } & \multicolumn{2}{|c|}{ Clase esqueletal tipo III } & \multirow[b]{2}{*}{$\mathbf{P}$} \\
\hline & Media & DS & Media & DS & \\
\hline \multicolumn{6}{|c|}{ Transversal } \\
\hline Punto A & (+)2,07 & $\pm 1,24$ & $(+) 3,59$ & $\pm 1,31$ & $0,0005^{*}$ \\
\hline Punto B & $(+) 4,37$ & $\pm 1,25$ & $(-) 3,68$ & $\pm 1,24$ & $0,0001 *$ \\
\hline Hioides & $(+) 3,13$ & $\pm 2,29$ & $(-) 1,22$ & $\pm 2,26$ & $0,0001 *$ \\
\hline \multicolumn{6}{|l|}{ Vertical } \\
\hline ENA & $(+) 0,97$ & $\pm 2,14$ & $(+) 1,12$ & $\pm 2,28$ & 0,71 \\
\hline Me & (+) 1,18 & $\pm 2,69$ & $(-) 0,81$ & $\pm 3,04$ & $0,025^{*}$ \\
\hline Hioides & $(+) 2,00$ & $\pm 2,17$ & $(-) 0,68$ & $\pm 2,56$ & $0,002^{*}$ \\
\hline
\end{tabular}

DS: desviación estándar. $\left(^{*}\right)$ indica diferencia estadística significativa. En mediciones transversal el signo (+) significa avance y el signo (-) significa retroceso. En la medición vertical el signo (+) significa ascenso y el signo (-) significa descenso. Ena: Espina nasal anterior; Me: Mentón.

\section{DISCUSION}

La cirugía para el cambio de imagen facial y para corregir las deformidades faciales, aumenta sostenidamente en los últimos años debido a la mejoría funcional y la mejoría de la morfología estética del rostro. Podrían representar el 5\% de la población y las indicaciones de estos procedimientos han variado desde los objetivos de los años 80 y 90 que eran principalmente para mejorar y estabilizar la oclusión hacia los objetivos más contemporáneos, orientados a mejorar la posición de las estructuras faciales, tratar enfermedades, mejorar la calidad de vida y contribuir a la estética facial (Posnick, 2014).

La morfología facial y las condiciones del rostro se relacionan con diversos factores tales como la ancestría, sexo, edad, índice de masa corporal, el tipo de trabajo y la exposición al sol. El crecimiento elevado del cráneo, orbita superior y huesos propios de la nariz son esenciales durante los primeros 5 años de vida (Fan et al., 2012); posteriormente, después de los 10 años de edad, se produce un gran desarrollo del tercio medio e inferior del rostro, promovido fundamentalmente por el crecimiento de la maxila y de la mandíbula (Ranly, 2000). De esta manera, las deformidades faciales se manifiestan habitualmente después de los 12 años de edad.

Estudios previos de nuestro grupo han evidenciado diferencias morfológicas entre sujetos con deformidad facial de clase II y clase III, donde sujetos de clase III presentan un mayor ancho facial y mayor ancho mandibular, pero semejante ancho maxilar, determinando la relevancia de la maxila, que puede presentar un crecimiento no proporcional a los otros huesos de la cara (Ravelo et al., 2020a).

El $92,2 \%$ de los sujetos tratados con cirugía ortognática presentan altos niveles de alteración funcional asociada a la deformidad facial (Eslamian et al., 2019). En términos de cirugía, la de tipo bimaxilar fue más frecuente en nuestra muestra, con el 90,1\% de los casos; aunque estudios como el de Eslamian et al. reportaron el 63\% de su muestra sometida a cirugía bimaxilar, es posible que la composición poblacional explique estas diferencias, considerando la mayor frecuencia de sujetos con clase II esqueletal y las complicaciones funcionales asociadas a ella. Casos más complejos requieren de mayores movimientos que se integran a los movimientos maxilar y mandibular más comunes (Goulart et al., 2020).

En la secuencia de 44 sujetos incluidos en esta investigación, se observó que los sujetos con deformidad de clase II y de clase III presentaron un adelantamiento de maxila en el $90 \%$ de los casos. El avance del complejo maxilar parece ser el más utilizado en sujetos clase II y puede ser asociado a deficiencias en el volumen de la vía aérea, para lo cual, los movimientos de avance y rotaciones anti horarias son los más habituales (Giralt-Hernando et al. 2021); de la misma forma, en sujetos de clase III, el avance maxilar puede ayudar a mejorar la vía aérea, proporciones estéticas y otros aspectos de la función del área 
(Giap et al., 2021); en la mayoría de los casos, el retroceso mandibular es realizado en este grupo de pacientes para optimizar el balance facial.

El tamaño de los movimientos generados fue en promedio de 2 a $3 \mathrm{~mm}$, lo cual genera impactos significativos en la apreciación de las proporciones faciales. Wadi et al. (2020) indicaron que la percepción de estética y atracción del perfil facial de sujetos de clase facial III fue significativamente más atractivo en la observación de las imágenes de proyección con cirugía ortognática que solo las imágenes de proyección con ortodoncia, determinando que el balance facial se obtiene con movimientos quirúrgico y no con movimientos ortodóncicos de camuflaje. De ese punto de vista, la magnitud del movimiento quirúrgico permite realizar el balance de la estructura facial.

En este sentido, la relación de tejidos blandos faciales es significativa y depende de los movimientos óseos (Olate et al., 2016; Bral et al., 2020) a los cuales se debe considerar en la estabilidad en el largo plazo. En esta investigación, la evaluación de corto plazo fue considerada en base a conocer el grado y tipo de movimiento generado, sin embargo, para evaluación de estabilidad de estos movimientos, variables como el grado de avance y el tipo de osteosíntesis tienen relevancia y deben ser considerados en las investigaciones (Sigua-Rodriguez et al., 2018; 2019).

Es posible concluir que existe una clara tendencia al avance maxilar, independiente de la deformidad facial. En sujetos con retrognatia mandibular, la OSRM es utilizada frecuentemente para el avance y en casos de prognatismo mandibular la OSRM es frecuentemente utilizada para retroceso. Los movimientos verticales son limitados.

RAVELO, V.; OLATE, G.; HUENTEQUEO-MOLINA, C.; HAIDAR, Z. S.; PARRA, M.; VÁSQUEZ, B. \&OLATE, S. Facial Morphology in Orthognathic Surgery. Is there a facial advance trend?. Int. J. Morphol., 39(2):1116-1122, 2021.

ABSTRACT: The objective of this research was to determine the preferred movements in the maxilla and mandible to obtain normality in facial morphology using superimposition techniques in 3D analysis. A descriptive study was carried out to evaluate bimaxillary bone displacement and hyoid bone in subjects facial class II and facial class III undergoing orthognathic surgery. were used as fixed points for superimposition: Nasion (N) - Silla (S) - Porion (Po) and the zygomatic-maxillary suture (Z). These points were superimposed in pre-surgical and postsurgical CBCT and was evaluated to displacement of the anterior nasal spine, Point A, Point B, Chin and the hyoid bone. For the evaluation and comparison of continuous variables before and after orthognathic surgery, was used the Student's t test. For the correlation between the variables, the Spearman test is used, considering a p value $<0.05$ as a significant difference. 44 subjects between 18 and 40 years old of both sexes were included in this research. A $90 \%$ of subjects a was performed a maxillay sagittal movement. The sagittal movement of mandibular advancement was performed in $100 \%$ with facial class type II, while $100 \%$ of the subjects with with facial class type III had a mandibular recession. The hyoid bone advanced in 26 of the 27 subjects with facial class type II. It is possible to conclude that there is a tendency for maxillary advancement, independent of facial deformity.

KEY WORDS: Orthognathic surgery; Facial morphology; Cephalometry; Bone movement.

\section{REFERENCIAS BIBLIOGRÁFICAS}

Bral, A.; Olate, S.; Zaror, C.; Mensink, G.; Coscia, G. \& Mommaerts, M. A prospective study of soft- and hard-tissue changes after mandibular advancement surgery: Midline changes in the chin area. Am. J. Orthof. Dentofacial Orthop., 157(5):662-7, 2020.

Eslamian, L.; Borzabadi-Farahani, A.; Badiee, M. R. \& Le, B.T. An objective assessment of orthognathic surgery patients. J. Craniofac. Surg., 30(8): 2479-82, 2019.

Fan, K.; Andrews, B.; Tabit, C. \& Bradley, J. The Sarnat studies in craniofacial biology. J. Craniofac. Surg., 23(1):37-43, 2012.

Giap, H. V.; Shin, J. W.; Chae, H. S.; Kim, Y. H.; Paeng, J. Y. \& Choi, H. W. Pharyngeal airway morphology in skeletal class III with mandibular asymmetry is improved after bimaxillary orthognathic surgery. J. oral Maxillofac. Surg., 79(5):1107-21, 2021.

Giralt-Hernando, M.; Valls-Ontañon, A.; Hass, Jr., O.L.; Masià-Gridilla, J. \& Hernandez-Alfaro, F. What are the surgical movements in orthognathic surgery that most affect the upper airways? A three-dimensional analysis. J. Oral Maxillofac. Surg., 79(2):450-62, 2021.

Goulart, D. R.; Huentequeo-Molina, C.; Alister, J. P. \& Olate, S. Threedimensional planning and surgical technique for modified Le Fort I and Le Fort III osteotomy in non-syndromic patients. J. Craniofac. Surg., 31(6):614-7, 2020.

Goulart, D. R.; Sigua-Rodriguez, E. A.; Fariña, R.; Olate, S. Condylar hiperplasia in a monozygotic twin girl: an argument about etiology. $J$. Craniofac. Surg., 29:599-602, 2018.

Mendelson, B. \& Wong, C. Changes in the facial skeleton with aging: Implications and clinical applications in facial rejuvenation. Aesthetic Plast Surg., 36(4):753-60, 2012.

Obwegeser, H. L.Orthognathic surgery and a tale of how three procedures came to be: a letter to the nest generations of surgeons. Clin. Plast. Surg., 34(3):331-55, 2007.

Olate, S.; Pozzer, L.; Unibazo, A.; Huentequeo-Molina, C.; Martinez, F. \& de Moraes M. LeFort I segmented osteotomy experience with piezosurgery in orthognathic surgery. Int. J. Clin. Exp. Med., 7(8):2092$5,2014$.

Olate, S.; Sigua, E.; Asprino, L. \& de Moraes, M. Complications in orthognathic surgery. J. Craniofac. Surg., 29(2):158-61, 2018.

Olate, S.; Zaror, C.; Blythe, J. N. \& Mommaerts, M. A systematic review of soft-to-hard tissue ratios in orthognathic surgery. Part III: Double jaw surgery procedures. J. Craniomaxillofac. Surg., 44(10):1599-606, 2016.

Posnick, J.C. Definition and prevalence of dentofacial deformities. In: Posnick, J. C., editor. Orthognathic surgery: principles and practice. St Louis, MO: Elsevier., 61-8, 2014.

Ranly, D. M. Craniofacial growth. Dent. Clin. North Am., 44(33):457-70, 2000. 
RAVELO, V.; OLATE, G.; HUENTEQUEO-MOLINA, C.; HAIDAR, Z. S.; PARRA, M.; VÁSQUEZ, B. \& OLATE, S. Morfología ósea facial en cirugía ortognática. ¿Existe tendencia hacia el avance facial?. Int. J. Morphol., 39(4):1116-1122, 2021.

Ravelo, V.; de Moraes, M. \& Olate, S. Relación transversal, vertical y sagital en sujetos con deformidad facial candidatos a cirugía ortognática. Int. J. Odontostomatol., 14(4):664-9, 2020a.

Ravelo, V.; Olate, G.; Moya, M. P.; Britto, L.; Garay, I. \& Olate, S. 3-D airway analysis related to facial morphology. Int. J. Morphol., 38(2): 423-6, 2020b.

Ravelo, V.; Sigua-Rodriguez, E.A.; Haidar, Z.S.; Brito, L.; Parra, M. \& Olate, S. Impact of facial bone deformity on nasal shape. Plast. Aesthet. Res., 8:19, 2021a.

Ravelo, V.; Olate, G.; Muñoz, G.; de Moraes, M. \& Olate, S. The airway volume related to the maxilla-mandibular position using $3 \mathrm{D}$ analysis. Biomed. Res. Int., 2021b. In press.

Shaw, R. B.; Katzel, E. B.; Koltz, P. F.; Kahn, D. M.; Puzas, E \& Langstein, H. Facial bone density: effects of aging and impact on facial rejuvenation. Aesthet Surg J., 32(8):937-42, 2012.

Sigua-Rodriguez, E. A.; de Medeiros, R. C.; Goulart, D. R.; BomfimAzevedo, V. J.; Olate, S. \& Albergaria-Barbosa, J. R. Comparative evaluation of different fixation techniques of the sagital split ramus osteotomy in $10 \mathrm{~mm}$ advancements: mechanical testing and screw insertion torque. J. Caniomaxillofac. Surg., 46(12):2082-7, 2018.

Sigua-Rodriguez, E. A.; Caldas, R. A.; Goulart, D. R.; de Moraes, P. H.; Olate, S.; Barao, V. A. \& Albergaria-Barbosa, J. R. Comparative evaluation of different fixation techniques for sagittal split ramus osteotomy in $10 \mathrm{~mm}$ advancement. Part two: Finite element analysis. J. Craniomaxillofac. Surg., 47(7):1015-9, 2019.

Sykes, J.; Riedler, K.; Cotofana. S. \& Palhazi, P. Superficial and deep facial anatomy and its implications for rhytidectomy. Facial Plast. Surg. Clin. N. Am., 28(3):243-51, 2020.

Villa, J.; Brito, L.; Parra, M.; Navarro, P.; de Moraes, M. \& Olate, S. Nasal septum desviation and inferior nasal concha bone hypertrophy in class III facial deformity. Int. J. Morphol., 38(6):1544-8, 2020.

Wadi, M. N. B.; Freitas, K. M. S.; Freitas, D. S.; Cançado, R. H.; de Oliveira, R. C. G.; de Oliveira, R. C.; Janson, G. \& Valarelli, F. P. Comparison of profile attractiveness between class III orthodontic camouflage and predictive tracing of orthognathic surgery. Int. J. Dent., 2020(11):7083940, 2020.

\author{
Dirección para correspondencia: \\ Dra. Bélgica Vásquez \\ Facultad de Ciencias de la Salud \\ Universidad de Tarapacá \\ Iquique \\ CHILE
}

E-mail: bvasquezp@uta.cl

Recibido: 26-03-2021

Aceptado: 04-05-2021 